\title{
Application of Multi-step Iterative ESN Method in Data Forecasting
}

\author{
Liu Shuang ${ }^{\text {a }}$, Sun Jianming \\ School of Computer and Information Engineering, Harbin University of Commerce, Harbin, 150028, China \\ ayscls2013@163.com
}

Keywords: Single-step prediction, multi-step direct prediction, multi-step iterative prediction, data prediction

\begin{abstract}
Aiming at the application of echo state network in the field of data prediction, the hierarchical structure and parameter configuration of echo state network are analyzed in detail. On this basis, the three processes of ESN single-step prediction, ESN multi-step direct prediction and ESN multi - step iterative prediction are constructed and compared with the experimental results of three different sequence data. Experimental results show that compared with ESN single-step prediction and ESN multi-step direct prediction, ESN multi-step prediction has higher prediction accuracy and more practical in the field of data prediction.
\end{abstract}

\section{Introduction}

The traditional prediction methods include linear Poisson model, regression model, Markov model and time series forecasting model [1-2]. Traditional predictive methods build models based on linear changes. The algorithm is simple and fast, and it can describe the linear time series data well. But in the era of large data, many data show the characteristics of non-linearity, autocorrelation, chaos, long correlation, suddenness and so on because of the increase of scale [3-4]. The traditional linear prediction method can not simulate the nonlinear time series well and is not suitable for the prediction of these data.

On this basis, there are machine learning algorithms such as neural networks and support vector machines, which are applied to network resource prediction [5]. Among them, the neural network prediction algorithm has a very good self-learning ability and nonlinear approximation ability [6]. By selecting different neural networks, the neural network algorithm is improved and processed, and the prediction performance of the neural network prediction algorithm is improved. Chan applied the Bayesian theory to the optimization of neural network dynamic parameters, and improved the accuracy of neural network prediction [7]. Larson combined the neural network prediction model with the gray model, and applied it to the network data forecasting, and achieved very good results [8]. Among the existing techniques applied to data prediction, the most mature and widely used is BP neural network prediction technology. BP neural network used gradient descent method for training, and achieved good prediction effect. But the algorithm still has many problems, including complex training methods, long time, low efficiency, and prediction results which are easy to fall into the local optimal solution.

In this paper, we use the applications of ESN in the field of data prediction as the core research content. By comparing ESN techniques such as single-step prediction, multi-step direct prediction and multi-step iterative prediction, a more reasonable ESN data prediction scheme is established.

\section{Process of multi-step prediction}

Let $X(n)$ be a time series, and the structure dimension of this time series is $m$, the time interval between each time node is $\Delta$, then the current time node data in $X(n)$ can be implemented according to the following mathematical formula: 


$$
x(n)=\{x(n-\Delta), x(n-2 \Delta), \cdots, x(n-(m-1) \Delta)\}^{T}
$$

The above relationship also reflects the dynamic change of time series data association. When the time series data update with time evolving, the current node after the first s time node data can be calculated using the following formula:

$$
x(n+s)=P\{x(n+s-\Delta), x(n+s-2 \Delta), \cdots, x(n+s-(m-1) \Delta)\}^{T}
$$

In the formula, $P$ denotes the mapping relationship between the previous time node data and (n+ s) time data, that is, the prediction function. The implementation of multi-step prediction is divided into two methods, one is the iterative method, and the other is the direct method. Among them, the prediction formula of the iterative method is as follows:

$$
x(n+s)=\underbrace{P(P(\cdots P(x(n))))}_{s}
$$

The essence of the iterative method is to perform $n$ times the results of single-step prediction. So the prediction accuracy of this method has a very large dependence on the accuracy of single-step prediction.

The prediction formula of the direct method is as follows:

$$
x(n+s)=P(x(n))
$$

The iterative method to perform multi-step prediction algorithm steps are as follows:

The first step is to configure the parameters of the pool in the ESN. That is, the pool size $N$, the spectral radius $R$, the input scaling factor $\delta_{I}$, and the sparsity degree $D$ are set, and initializing settings are performed for the weight connection matrices $\Phi_{i c}$ and $\Phi_{c c}$.

In the second step, the input-output relationship of the ESN is reconstructed as shown in equation (5).

$$
\left\{\begin{array}{l}
i(n)=P(x(n-\Delta), x(n-2 \Delta), \cdots, x(n-(m-1) \Delta)) \\
o_{p}(n)=x(n+1)
\end{array}\right.
$$

In the formula, $i(n)$ is the input of $\operatorname{ESN}, o_{p}(n)$ is the output prediction of $\operatorname{ESN}$ and $x(n)$ is the intermediate variable in the reconstruction process.

The third step is to perform training on the ESN after deconstructing the input-output relationship. After judging that the ESN has converged, record the configuration of $\Phi_{c o}$.

The fourth step, calculate the single step output based on the ESN model and $\Phi_{c o}$, as shown in equation (6).

$$
\hat{o}(n+1)=\Phi_{c o} i(n)
$$

In the fifth step, the single-step calculation result is used as the deconstructed data, as shown in formula (7).

$$
x(n)=\hat{o}(n+1)
$$

In the sixth step, the operations of the fourth step and the fifth step are repeatedly executed s times until the specific data $\hat{o}(n+s)$ of the predicted output is obtained.

The multi-step forecasting process of the direct method and the iteration method are basically the same. Only in the output prediction of the implementation of the calculation is different. The multi-step predictive calculation of the direct method is shown in the equation (8).

$$
\hat{o}(n+s)=\Phi_{c o} i(n)
$$




\section{Experimental results and analysis}

In order to ensure that the ESN constructed in this paper can obtain the desired effect, it is necessary to configure the key parameters reasonably. Considering the number of neurons in the input layer and the output layer are 8 and 3 respectively, the size of the whole ESN is not very large. Therefore, the number of neurons is 80 . To ensure that the ESN exhibits a stable echo state, set the spectral radius to a magnitude of 0.75 . The sparsity parameter is set to $10 \%$ in order to minimize the degree of coupling of the ESN pool as much as possible. Considering that the input neuron data and the output neuron data are at the same number level, set the input scaling factor $\delta_{I}$ to 0.4.

In Figure 1, there are four curves for the comparison. Where FM represents the true value curve of FM sequence data, MPO represents the ESN single-step prediction curve, MPD represents the ESN multi-step direct prediction curve, and OP represents the ESN multi-step iterative prediction curve.

From the comparison of four curves, ESN multi-step iterative prediction is the best and the error is the least. The error of ESN multi-step prediction is more than that of ESN multi-step iterative prediction method. ESN single step prediction error is the largest, and there are large fluctuations.

Next, we further investigate the prediction of SYXX sequence data and WGSJ sequence data under three methods, and the prediction results are shown in Fig. 5 and Fig. 6, respectively.

In Figure 2, SYXX represents the true value of the SYXX sequence data. In Figure 3, WGSJ represents the true value of the WGSJ sequence data. MPO curve, MPD curve and OP curve represent ESN single-step prediction, ESN multi-step direct prediction and ESN multi-step iterative prediction respectively.

From the results of Fig. 2 and Fig. 3, the ESN multi-step iterative prediction method also shows the characteristics of stable prediction and small prediction error.
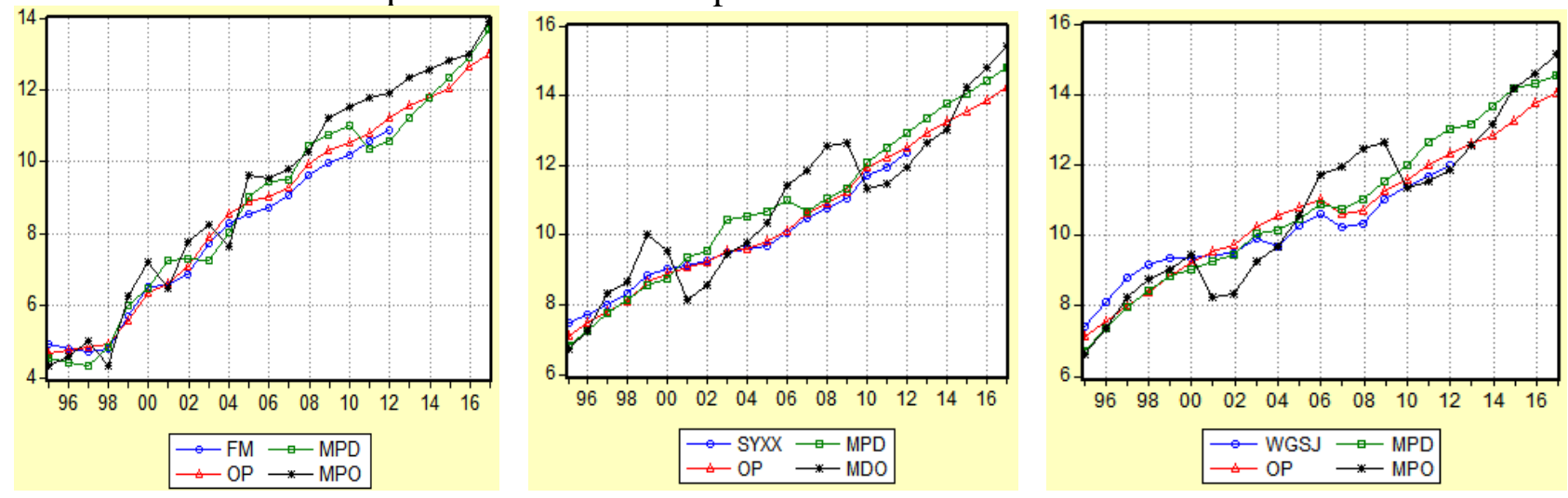

Figure 1 Prediction results of FM Figure 2 Pprediction results of SYXX Figure.3 Prediction results of WGSJ

The quantitative results of the three methods are investigated. Here, the prediction process of the FM sequence data, SYXX sequence data, and WGSJ sequence data is quantitatively compared with the prediction accuracy of the four indicators, which are root mean square error $\delta_{\text {rmse }}$, average absolute error $\delta_{\text {mad }}$, average percentage error $\delta_{\text {mape }}$, and square root error $\delta_{\text {nrmse }}$. The results are shown in Table 1.

It can be seen from the results in Table 2 that ESN multi-step iterative prediction is more accurate than ESN single-step prediction and ESN multi-step direct prediction on the four prediction accuracy indexes for the three sets of sequence data prediction results. 
Table 1 Comparison of the results of three methods of prediction accuracy

\begin{tabular}{c|c|c|c|c}
\hline \multirow{2}{*}{ Data } & $\begin{array}{c}\text { Accuracy } \\
\text { index }\end{array}$ & OP prediction & $\begin{array}{c}\text { MPD } \\
\text { prediction }\end{array}$ & $\begin{array}{c}\text { MPO } \\
\text { prediction }\end{array}$ \\
\hline \multirow{4}{*}{$\begin{array}{c}\text { FM sequence } \\
\text { data }\end{array}$} & $\delta_{\text {rmse }}$ & 0.443 & 0.782 & 0.904 \\
\cline { 2 - 5 } & $\delta_{\text {mad }}$ & 0.459 & 0.801 & 0.913 \\
\cline { 2 - 5 } & $\delta_{\text {mape }}$ & $4.93 \%$ & $10.03 \%$ & $11.89 \%$ \\
\hline \multirow{4}{*}{$\begin{array}{c}\text { SYXX } \\
\text { sequence data }\end{array}$} & $\delta_{\text {rrmse }}$ & 0.059 & 0.096 & 0.096 \\
\cline { 2 - 5 } & $\delta_{\text {rmse }}$ & 0.545 & 0.936 & 1.128 \\
\cline { 2 - 5 } & $\delta_{\text {mad }}$ & 0.552 & 0.944 & 1.135 \\
\hline \multirow{3}{*}{$\begin{array}{c}\text { WGSJ } \\
\text { sequence data }\end{array}$} & $\delta_{\text {mape }}$ & $4.86 \%$ & $10.27 \%$ & $11.86 \%$ \\
\cline { 2 - 5 } & $\delta_{\text {nrmse }}$ & 0.116 & 0.169 & 0.198 \\
\cline { 2 - 5 } & $\delta_{\text {rmse }}$ & 0.538 & 1.022 & 1.217 \\
\cline { 2 - 5 } & $\delta_{\text {mad }}$ & 0.574 & 1.085 & 1.236 \\
\hline
\end{tabular}

\section{Conclusion}

Aiming at the problem of data prediction, the research on echo state network prediction method is studied deeply. First, a three-level model of echo state network is given. The mathematic model of the input layer, the hidden layer and the output layer neuron and the mathematical model of the relationship between the layers are expounded. Secondly, the four key parameters of the echo state network are analyzed, i.e. the size of the pool, the spectral radius, the input scaling factor and the sparse degree. Thirdly, ESN single-step prediction process and ESN multi-step prediction process are constructed. The mathematical model of ESN prediction is described from single-step prediction, multi-step direct prediction and multi-step iterative prediction. Experimental studies are carried out on three different sets of sequence data. The experimental results show that ESN multi-step iterative prediction has higher prediction precision and can predict the future changes of sequence data effectively.

\section{Acknowledgments}

This study was supported by Youth Innovative Talent Training Plan Project in Harbin University of Commerce (17XN005).

\section{References}

[1] Shu-wei Ren, Xiao-feng Ju. Empirical study on discontinuous innovation affected by technology policy of Beijing [J]. Information Technology Journal, 12(1): 162-167. (2013)

[2] Aghion Philippe, P Howitt. A model of growth through creative destruction [J]. Economet rica, 60(2): 323-351. (2012)

[3] Bjorg Olafsdottir Kristin, Schulz Michael, Mudelsee Manfred. REDFIT-X: Cross-spectral analysis of unevenly spaced paleoclimate time series [J]. Computers and Geosciences, 91: 11-18. (2016)

[4] Zhu Xiao-qin, Ma Lu. The influence of enterprise network capability on technology innovation moderating role of learning orientation [J]. Proceedings of 2013 4th International Asia Conference on 
Industrial Engineering and Management Innovation, IEMI 2013, 311-318.(2013)

[5] Nand Ravneil, Chandra Rohitash. Reverse neuron level decomposition for cooperative neuro-evolution of feedforward networks for time series prediction[C]. Australasian Conference on Artificial Life and Computational Intelligence, 9592: 171-182. (2016)

[6] Suorez D. Persistence of innovation in unstable environments: continuity and change in the firm's innovative behavior [J]. Research Policy, 43(4): 726-736.(2014)

[7] Chan J, Lam C, Boris C. An innovative financial time series model: the geometric process model [J]. Advances in Intelligent Systems and Computing, 251: 81-99.(2014)

[8] Larson J, Park J. From developmental to network state: government restructuring and ICT-led innovation in Korea [J]. Telecommunications Policy, 38(4): 344-359.(2014) 